\title{
Efficient Least-Squares 2-D-Cubic Spline for Concurrent Dual-Band Systems
}

\author{
Naveen Naraharisetti, Member, IEEE, Patrick Roblin, Senior Member, IEEE, \\ Christophe Quindroit, and Shahin Gheitanchi, Member, IEEE
}

\begin{abstract}
This paper presents and compares two types of 2-D cubic-spline (2-D-CS) digital predistorters for linearizing a power amplifier (PA), which is used in dual-band transmitters. In the conventional 2-D-CS representation, the gain functions must be first extracted using an alternate basis, whereas in the proposed 2-D least-squares cubic-spline (2-D-LSCS) approach, a new 2-D-CS basis is introduced such that the basis weights can be extracted directly from the measured data using the least-squares method. The 2-D basis functions are calculated from 1-D basis functions to reduce the signal-processing resource usage in the real-time implementation. A field-programmable gate-array (FPGA) test bench integrating the concurrent dual-band RF system is utilized to verify the linearization performance of the new 2-D-LSCS predistorter. Two different test scenarios involving three carrier-wideband code division multiple access and long-term evolution signals, which are $310 \mathrm{MHz}$ apart, are considered. The experimental results on a $10-\mathrm{W}$ dual-band PA shows that the proposed 2-D-LSCS basis improves the performance up to $3 \mathrm{~dB}$ in both the adjacent channel power ratio and normalized mean square error with reduced FPGA resources and faster extraction time when compared to the conventional 2-D memory-polynomial and 2-D-CS approaches.
\end{abstract}

Index Terms-Concurrent bands, digital predistortion (DPD), power amplifier (PA), 2-D lookup table (2-D LUT), 2-D cubic spline (2-D-CS).

\section{INTRODUCTION}

$\mathbf{H}$ IGH DATA-RATE transmissions employ complex envelop techniques like WCDMA and orthogonal frequency division multiplexing (OFDM) signals for their spectral efficiency. However, these modulation schemes impose strict linearity requirements on the power amplifier (PA) because of their nonconstant envelope with high peak to average power

Manuscript received May 18, 2014; revised August 15, 2014, November 17, 2014, and April 08, 2015; accepted May 09, 2015. Date of publication June 12, 2015; date of current version July 01,2015 . This work was supported by the Altera Corporation-Wireless Systems Solutions Group and by the National Science Foundation (NSF) under Grant ECS 1129013.

N. Naraharisetti was with the Department of Electrical and Computer Engineering, The Ohio State University, Columbus, OH 43210 USA. He is now with GatesAir Inc., Mason, OH, USA (e-mail: naraharn@ece.osu.edu).

P. Roblin is with the Department of Electrical and Computer Engineering, The Ohio State University, Columbus, OH 43210 USA (e-mail: roblin@ece. osu.edu).

C. Quindroit was with the Department of Electrical and Computer Engineering, The Ohio State University, Columbus, OH 43210 USA. He is now with LPA Concepts, 33650 Martillac, France (e-mail: quindroc@ece.osu.edu).

S. Gheitanchi is with Altera Europe, Buckinghamshire HP12 4XF, U.K. (e-mail:sgheitan@altera.com).

Color versions of one or more of the figures in this paper are available online at http://ieeexplore.ieee.org.

Digital Object Identifier 10.1109/TMTT.2015.2435731

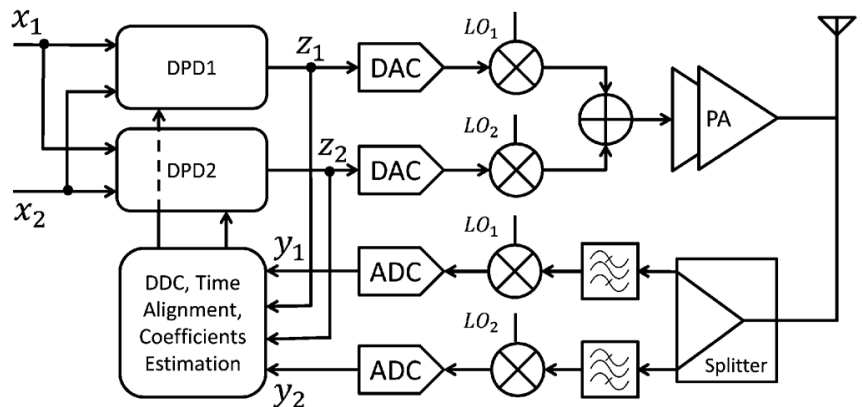

Fig. 1. General architecture of concurrent dual-band DPD system.

ratio (PAPR). Due to the nonlinearities of PAs, the signal develops spectral regrowth and intermodulation distortion (IMD). A linearizing technique is needed to reduce the spectral regrowth and pass the signal through the mask requirements imposed by the Federal Communications Commission (FCC), while achieving a good power efficiency. Digital predistortion (DPD) is one of the commonly used linearizing techniques because of its robustness, moderate implementation cost, and high accuracy.

With the current advancements in the design of broadband PAs operating from several megahertz to several gigahertz, new transmitter architectures are being deployed using a single PA accommodating concurrent bands to reduce the cost [1], [2]. These advancements impose challenges on the DPD implementation, which requires sampling rates of 5-7 times the signal bandwidth to account for in-band and out-of-band IMDs. Digital-to-analog converters (DACs) and analog-to-digital converters (ADCs) operating at such high sampling rates are not cost effective. A frequency-selective technique can be incorporated into the DPD to compensate for the in-band and interband IMDs [3], and also to relax the sampling rate requirement for both the ADC and DAC. The general architecture for the concurrent dual-band system with separate paths for each band defining the frequency-selective technique is shown in Fig. 1.

The complexity of the DPD system can be further reduced by transmitting widely separated signals, as the outerband IMDs are far from the two data bands. These IMDs can be easily filtered by placing a transmit filter at the output of the PA, which need not have a high skirt selectivity. Although the filter removes the outerband distortion, the in-band distortion by the current band and also from the concurrent band will still need to be compensated by the DPD system [4], [5]. 
There are a number of DPD techniques developed for the concurrent dual-band system in the prior literature. A frequency-selective technique was initially introduced to compensate for the in-band, cross-modulation, and interband IMDs [3]. Since the technique was limited by the characterization accuracy and also did not include a time-selective memory effect in the extraction process, [6] reported a 2-D DPD model using memory polynomials (MPs). As will be discussed in Section II, polynomials may not always be best suited for a highly nonlinear device or highly saturated PA. In [1], a 1-D lookup table (LUT) model is developed for the concurrent dual-band system. Although the implementation cost is reduced by using a 1-D LUT, the linearization performance is limited because of excluding the cross-modulation products. The 2-D DPD model in [6] is rearranged in [7] to use 1-D LUTs instead of 2-D LUTs, but the performance is still limited by the 2-D-DPD model. The number of coefficients in the 2-D-DPD model can be reduced by implementing a twin nonlinear two-box model in which a static nonlinearity is implemented in the first box and a lower nonlinearity order MP model is used in the second box [8]. A new 2-D quasi-exact inverse (2-D-QEI) [9] of a PA model based on a direct learning architecture is used to improve the performance of the DPD in the presence of additive noise at the PA output. Most of the algorithms and architectures that were developed in the literature rely on a single polynomial for the entire range of the input in each band.

In this paper, a piece-wise polynomial model is adopted because of its advantageous local stability over regular polynomials, as discussed in Section II [10]. Two methods, 2-D cubic spline (2-D-CS) and 2-D least-squares cubic spline (2-D-LSCS), are presented for the extraction of 2-D cubic-spline (C-spline) representations. In the conventional C-spline approach (i.e., 2-D-CS), an external method must be used for a preliminary extraction of the gain functions to permit their evaluation at the knots for the $\mathrm{C}$-spline extraction [5]. This reliance on an alternate extraction function is a serious limitation, as the $\mathrm{C}$-spline representation cannot be expected to outperform the original extraction technique in terms of linearization. In this paper, a new 2-D-CS basis is initially developed for the new 2-D-LSCS, and then the weight coefficients for these 2-D-CS basis expansion can be directly extracted from the measured data using the least-squares (LS) method.

The new 2-D-LSCS is found to achieve better performance than a 2-D memory-polynomial (2-D-MP) and 2-D-CS model, while requiring reduced extraction time due to the reduced number of coefficients and reduced polynomial order of the C-spline. Thus, the new LSCS model could also be utilized in the above-mentioned architectures and further reduce the field-programmable gate-array (FPGA) resources.

Although there is reduction in the extraction time, it is also important to minimize the real-time signal-processing resources required to calculate the 2-D basis functions (polynomials or piece-wise polynomials). In this paper, an architecture similar to [11] is used as a compromise between the usage of DSP blocks and memory. The same resource-saving architecture will be used here for both the 2-D-LSCS and 2-D-MP for fair comparison. However, the 2-D-LSCS will require a substan- tially reduced amount of FPGA/DSP resources compared to the 2-D-MP basis due to the reduced number of coefficients it relies upon.

This paper is organized as follows. Section II presents the advantages of piece-wise polynomials over regular polynomials. In Section III, the two types of extraction for the 2-D-CS representation are presented and compared. The experimental measurement results are then reported in Section IV to compare the performance of these two methods with a conventional polynomial model. Finally, the results obtained for the new proposed 2-D-CS linearization are summarized in Section V.

\section{Piece-Wise Polynomials}

A 2-D-MP model of limited order is commonly used to represent the gains of a PA for the entire input envelope range [6], [12], [13]. A polynomial expansion with higher order is required to represent PAs that are highly nonlinear and saturated. However, extracting such a polynomial-based model can lead to a numerically ill-conditioned regression matrix and yields to a highly oscillatory solution when the data is sparse [10]. Due to this ill conditioning, the coefficients are extremely sensitive to the data [14], thereby forcing to update the system even if there is a small error in reading at input data. The coefficients also have a global scope, which tend to effect the entire curve and thus increase the error. A piece-wise polynomial representation like the $\mathrm{C}$-spline may be considered instead of regular polynomials to remediate these issues.

It may become profitable to rely on piece-wise polynomials of low-order rather than high-order polynomials for the entire envelope range to provide a more robust representation. Since lower order polynomials are used, they do not have oscillations like the Runge phenomenon [15], [16]. The condition number of the matrix created using piece-wise polynomials will also be smaller than the one using regular polynomials, thereby making the system more stable to errors at the input. The C-spline coefficients have a local scope, i.e., when an approximate value is used for a particular knot, the error introduced will only create a local distortion in the shape of the spline, which does not propagate to the entire input range [17]. As a result, a more robust extraction is expected for linearizing subsequent data after the training is performed.

\section{2-D-CSs}

When amplifying narrowband signals, the PAs can be assumed to be memoryless and thereby a memoryless DPD can be used to linearize them. However, this assumption is no longer valid for wideband communication signals that are currently in use. Implementing DPD, which takes into account time-selective memory effects, gives a considerable improvement in the adjacent channel power ratio (ACPR) and normalized mean square error (NMSE) [10]. A generalized frequency- and timeselective memory model, used to represent the output of the PA in each band after the out-of-band IMDs are filtered, is as follows:

$$
y_{i}(n)=\sum_{m=0}^{M-1} G_{i}^{(m)}\left(\left|x_{1}(n-m)\right|^{2},\left|x_{2}(n-m)\right|^{2}\right) x_{i}(n-m)
$$




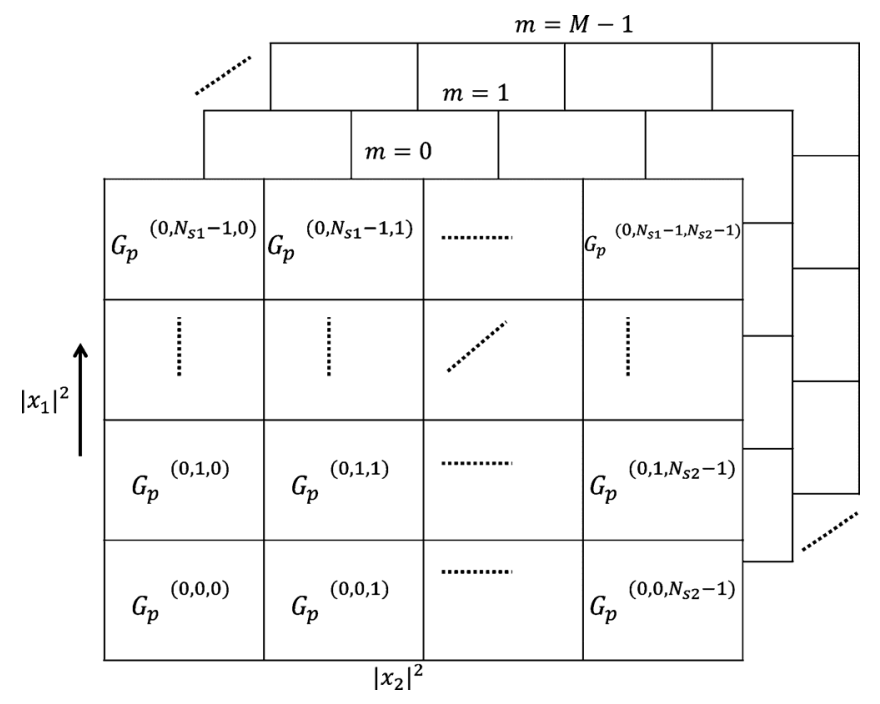

Fig. 2. Graphical representation of the gains $G_{p}^{(m, u, v)}\left(\left|x_{1}\right|^{2},\left|x_{2}\right|^{2}\right)$ as a function of $\left|x_{1}\right|^{2}$ and $\left|x_{2}\right|^{2}$ for all memory delay indices $m$.

where $\left|x_{1}(n-m)\right|^{2}$ and $\left|x_{2}(n-m)\right|^{2}$ are the envelope squares of each frequency band with memory and a memory depth of $M, i \in[1,2] . G_{1}^{(m)}\left(\left|x_{1}\right|^{2},\left|x_{2}\right|^{2}\right)$ and $G_{2}^{(m)}\left(\left|x_{1}\right|^{2},\left|x_{2}\right|^{2}\right)$ are the gain terms, which are a function of the envelop squares of both the bands for the memory delay index $m$.

These gain functions can be represented using different basis functions such as conventional polynomials [6], orthogonal polynomials [14], or splines[5]. In this paper, 2-D-CSs are used to represent these gains. Two methods are developed to design a 2-D-CS DPD, and their performance and limitations are compared.

\section{A. 2-D Conventional C-Spline}

1) Extraction: The generation of a 2-D-CS starts with the synthesis of multiple 1-D C-splines, which are then extended to 2-D. A 1-D C-spline consists of piece-wise polynomials of order 3, which are not only continuous for the polynomials at the knots where the splines connect, but also for their first and second derivatives [5], [15]. Furthermore, for $N_{s}+1$ knots, $N_{s}$ splines are defined with $4 N_{s}$ coefficients. $N_{s}-1$ equations comes from the continuity of polynomials, the first and second derivative, respectively. $N_{s}+1$ equations comes from the fact that splines needs to pass through these knots. In total there are $4 N_{s}-2$ equations, but there are $4 N_{s}$ unknowns from the $N_{s}$ splines. Since this is under-determined system, two other conditions need to be imposed on the system to generate unique cubic splines. A natural cubic spline is used in this paper so that the second derivative is set to zero at the end points. However, other conditions are possible like parabolic runout (the second derivative of the end points is equal to the second derivative of the neighbors), and cubic runout (second derivative of the end points are equal to combination of the two neighbors [15]).

The 2-D gain function for different memory delay indices $m$ is shown in Fig. 2 with $G_{p}^{(m, u, v)}\left(\left|x_{1}\right|^{2},\left|x_{2}\right|^{2}\right)$ representing the 2-D-CS for the particular region defined by indices $(m, u, v)$. The entire 2-D space is divided into a grid-like structure with the grid intersections representing the knots $(u, v)$. The number of splines along each axis of $\left|x_{1}\right|^{2}$ and $\left.\left|x_{2}\right|^{2}\right)$ is defined as $N_{s 1}$ and $N_{s 2}$.

The gain values at the knots are calculated using the MP model (2),

$$
\begin{aligned}
x_{1}(n)= & \sum_{m=0}^{M-1} \sum_{k=0}^{N-1} \sum_{j=0}^{k} d_{1 k j}^{(m)}\left|y_{1}(n-m)\right|^{2 j} \\
& \cdot\left|y_{2}(n-m)\right|^{2(k-j)} y_{1}(n-m) \\
x_{2}(n)= & \sum_{m=0}^{M-1} \sum_{k=0}^{N-1} \sum_{j=0}^{k} d_{2 k j}^{(m)}\left|y_{2}(n-m)\right|^{2 j} \\
& \cdot\left|y_{1}(n-m)\right|^{2(k-j)} y_{2}(n-m)
\end{aligned}
$$

where $y_{1}(n)$ and $y_{2}(n)$ are the sampled received signals, $x_{1}(n)$ and $x_{2}(n)$ are the sampled input signals, $d_{1 k j}^{(m)}$ and $d_{2 k j}^{(m)}$ are the coefficients of the MP, and $\left|y_{1}(n)\right|$ and $\left|y_{2}(n)\right|$ are the absolute values of complex envelops for the lower and upper bands, respectively.

The construction of these 2-D-CSs is performed as follows.

a) Firstly, start with the extraction of 1-D C-splines along the $\left|x_{1}\right|^{2}$ axis, as shown in Fig. 2, so that the gain function in (1) can be written for a given memory delay index $m$ and frequency bands $p, q \in[1,2]$ and $p \neq q$ as follows:

$$
\begin{aligned}
G_{p}^{(m, u, v)}\left(\left|x_{1}\right|^{2},\left|x_{2}\right|^{2}\right)= & \sum_{i=0}^{3} b_{p i}^{(m u v)}\left(\left|x_{q}\right|^{2}\right)\left(\left|x_{p}\right|^{2}-\left|x_{p, u}\right|^{2}\right)^{i} \\
& \forall u \in\left[0, N_{s 1}-1\right] ; \quad \forall v \in\left[0, N_{s 2}-1\right] ; \\
& \forall m \in[0, M-1]
\end{aligned}
$$

where $G_{p}^{(m, u, v)}\left(\left|x_{1}\right|^{2},\left|x_{2}\right|^{2}\right)$ is the gain function represented by a 2-D-CS for the intersecting area spanned by $[u, u+1]$ along $\left|x_{1}\right|^{2}$ and $[v, v+1]$ along $\left|x_{2}\right|^{2}$, as shown in Fig. 2. $\left|x_{p, u}\right|^{2}$ are the knot values along the $\left|x_{p}\right|^{2}$ axis given by $\left[\left|x_{p, 0}\right|^{2}\left|x_{p, 1}\right|^{2} \ldots\left|x_{p, N_{s 1}}\right|^{2}\right]$. The coefficients $b_{p i}^{(m u v)}\left(\left|x_{q}\right|^{2}\right)$, which are extracted in this step for all the splines $i$ along $\left|x_{p}\right|^{2}$, are a function of the $\left|x_{q}\right|^{2}$ parameter.

b) Next, 1-D C-splines are extracted for the extracted coefficients in (3) along $\left|x_{q}\right|^{2}$. It can be observed that the coefficients $b_{p i}^{(m u v)}\left(\left|x_{q}\right|^{2}\right)$ from (3) are used to construct the 1-D C-splines in (4),

$$
b_{p i}^{(m u v)}\left(\left|x_{q}\right|^{2}\right)=\sum_{j=0}^{3} a_{p i j}^{(m u v)}\left(\left|x_{q}\right|^{2}-\left|x_{q, v}\right|^{2}\right)^{j}
$$

where $\left|x_{q, v}\right|^{2}$ are the knot values along the $\left|x_{2}\right|^{2}$ axis, given by $\left[\left|x_{q, 0}\right|^{2} \quad\left|x_{q, 1}\right|^{2} \ldots\left|x_{q, N_{s 2}}\right|^{2}\right] . a_{p i j}^{(m u v)}$ are the $\mathrm{C}$-spline coefficients for the intersecting knot area spanned by $[u, u+1]$ along $\left|x_{1}\right|^{2}$ and $[v, v+1]$ along $\left|x_{2}\right|^{2}$.

c) Finally the 2-D-CS can be constructed by substituting (4) in (3) as follows:

$$
\begin{array}{r}
G_{p}^{(m, u, v)}\left(\left|x_{1}\right|^{2},\left|x_{2}\right|^{2}\right)=\sum_{i=0}^{3} \sum_{j=0}^{3} a_{p i j}^{(m u v)}\left(\left|x_{p}\right|^{2}-\left|x_{p, u}\right|^{2}\right)^{i} \\
\cdot\left(\left|x_{q}\right|^{2}-\left|x_{q, v}\right|^{2}\right)^{j}
\end{array}
$$




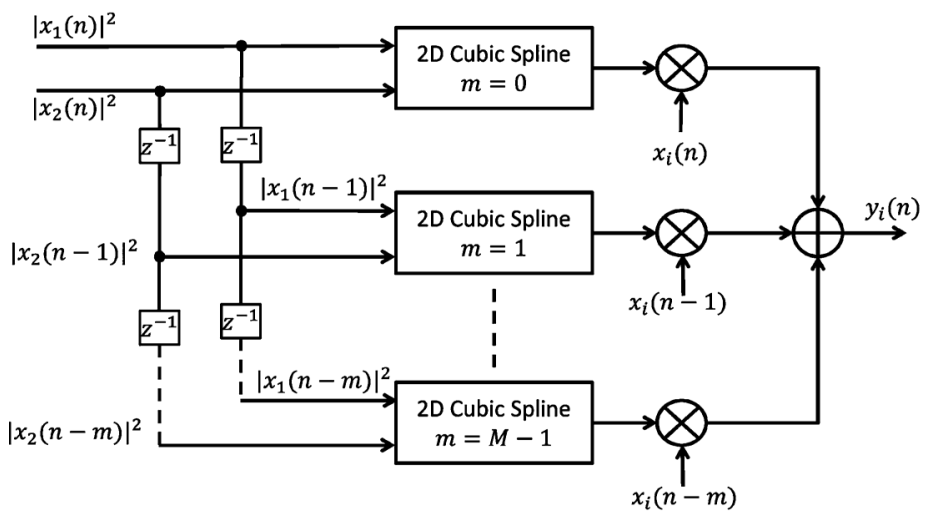

(a)

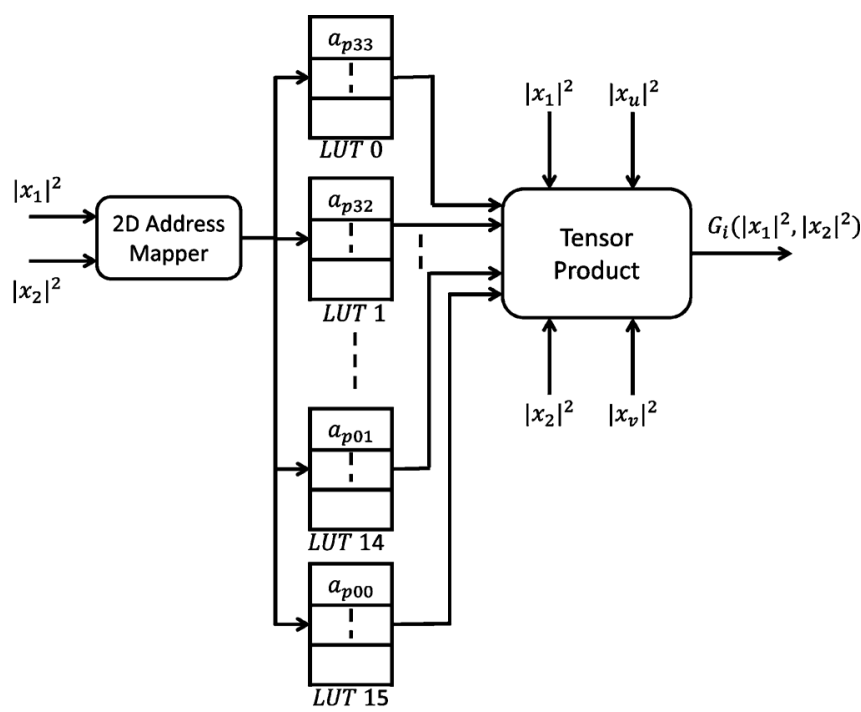

(b)

Fig. 3. Implementation of 2-D-CS with memory for each band $i$. (a) Memory cubic spline. (b) 2-D-CS block.

The result is equivalent to a tensor product of univariate $\mathrm{C}$-spline interpolation, as shown in (5).

2) Implementation: The architecture for the 2-D-CS is shown in Fig. 3(a), and the implementation of the C-spline in each memory delay branch is shown in Fig. 3(b). Each branch corresponds to a memory delay index $m$ to take into account the time-selective memory effect of the PA. The extracted coefficients can be stored in a 2-D-LUT structure, as shown in Fig. 3(b). $a_{p i j}^{(m u v)}$ in (5) are the coefficients of the 2-D-CS, which are used to interpolate the gain values for the intersecting knot area spanned by $[u, u+1]$ along $\left|x_{1}\right|^{2}$ and $[v, v+1]$ along $\left|x_{2}\right|^{2}$ for each memory index $m$.

To implement the 2-D-LUT, the entire LUT structure can be divided into a sequence of multiple 1-D-LUTs, as shown in Fig. 3(b). Each coefficient of the cubic polynomial is stored in separate 1-D-LUTs and the address for each coefficient is calculated based on the values of $\left|x_{1}(n-m)\right|^{2}$ and $\left|x_{2}(n-m)\right|^{2}$. The normalized envelope square of each band is multiplied with the number of splines to get the address along each axis. The effective 2-D address is calculated using these values, as shown in (6),

$$
\begin{aligned}
\operatorname{addr}_{1} & =\text { floor }\left\{\left|x_{1}(n-m)\right|^{2} \cdot N_{s 1}\right\} \\
\operatorname{addr}_{2} & =\text { floor }\left\{\left|x_{2}(n-m)\right|^{2} \cdot N_{s 2}\right\} \\
\operatorname{addr}_{a_{p i j}} & =\operatorname{addr}_{2}+\operatorname{addr}_{1} \cdot N_{s 2}
\end{aligned}
$$

where $\operatorname{addr}_{1}$ and $\operatorname{addr}_{2}$ defines the address in each direction, respectively, and $\operatorname{addr}_{a_{p i j}}$ is the compounded 2-D address for the 2-D-CS coefficients $a_{p i j}^{(m u v)}$ for any arbitrary $\left|x_{1}(n-m)\right|^{2}$ and $\left|x_{2}(n-m)\right|^{2}$ values. The coefficients can be selected from the LUT using this address and can be used to calculate the respective gains $G_{p}^{(m, u, v)}\left(\left|x_{1}(n-m)\right|^{2},\left|x_{2}(n-m)\right|^{2}\right)$ using (5). The floor function is effectively implemented by using the correct number of most significant bits in the fixed point notation, thereby the address mapper just requires a single multiplier and single adder.
A major limitation of using a 2-D-CS is the necessity to first evaluate the gains $G_{p}^{(m, u, v)}\left(\left|x_{1}\right|^{2},\left|x_{2}\right|^{2}\right)$ at the knots $\left(\left|x_{1, u}\right|^{2},\left|x_{2, v}\right|^{2}\right)$ using a separate modeling method before proceeding to the extraction of the C-spline coefficients $a_{p i j}^{(m u v)}$. The $\mathrm{C}$-spline coefficients must indeed be extracted at the knots to enforce exactly the continuity up to second-order derivatives. Note also that this model requires $2 M \cdot N_{s 1} \cdot N_{s 2} \cdot 16$ coefficients, which grows rapidly with an increase in the number of splines along each axis.

\section{B. 2-D-LSCS}

In 2-D-CS, the gain values at the knots must first be computed to perform the spline extraction since the measured input data are not usually located at the knots. Hence, an auxiliary model, in this case, a 2-D-MP model, is used to fit the data and extract the gain values at the required knots. The linearization performance is then limited by this auxiliary model rather than the characteristics of the $\mathrm{C}$-spline function. Furthermore, this method also requires a large number of coefficients. To overcome these deficiencies, a new $\mathrm{C}$-spline basis representation is developed in this section for which the coefficients can be extracted directly from the measured data using the LS method. This C-spline basis is designed to exhibit the desired local focus discussed in Section III-A while also being sensitive to the data distribution [18] via the selection of a nonuniform knot distribution.

1) Extraction: The gain function for each memory delay index $m$ in (1) can be represented using the following new 2-D basis functions $\phi_{i j}\left(\left|x_{1}\right|^{2},\left|x_{2}\right|^{2}\right)$ as

$$
\begin{aligned}
& G_{1}^{(m)}\left(\left|x_{1}\right|^{2},\left|x_{2}\right|^{2}\right)=\sum_{i=0}^{N_{s 1}} \sum_{j=0}^{N_{s 2}} c_{1 i j}^{(m)} \phi_{i j}\left(\left|x_{1}\right|^{2},\left|x_{2}\right|^{2}\right) \\
& G_{2}^{(m)}\left(\left|x_{1}\right|^{2},\left|x_{2}\right|^{2}\right)=\sum_{i=0}^{N_{s 1}} \sum_{j=0}^{N_{s 2}} c_{2 i j}^{(m)} \phi_{i j}\left(\left|x_{1}\right|^{2},\left|x_{2}\right|^{2}\right)
\end{aligned}
$$




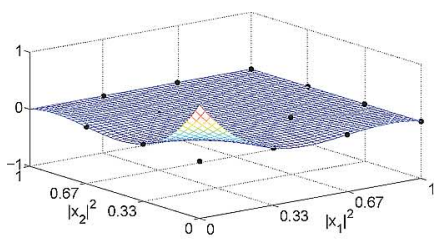

(a)

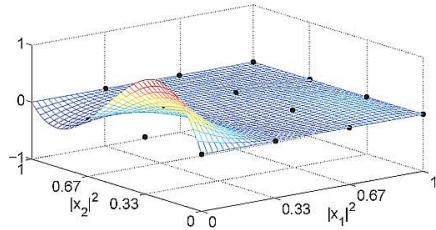

(e)

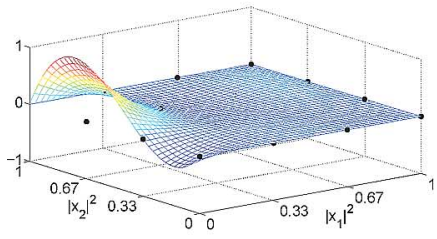

(i)

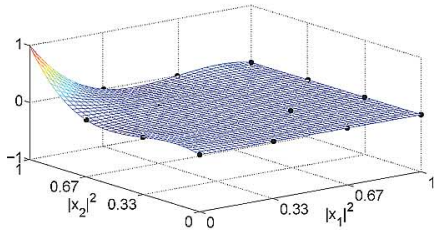

(m)

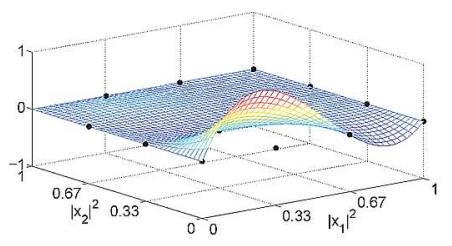

(b)

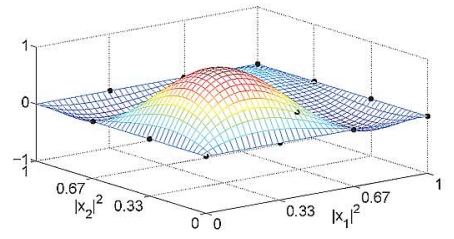

(f)

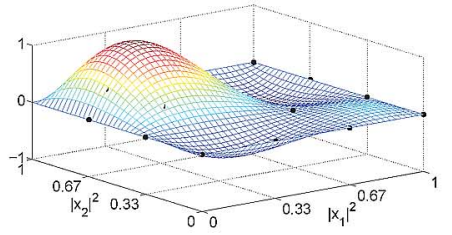

(j)

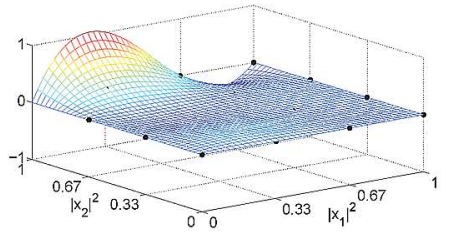

(n)

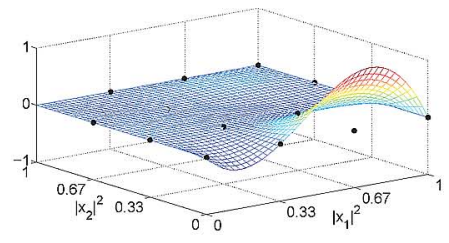

(c)

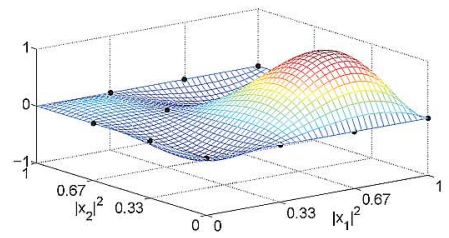

(g)

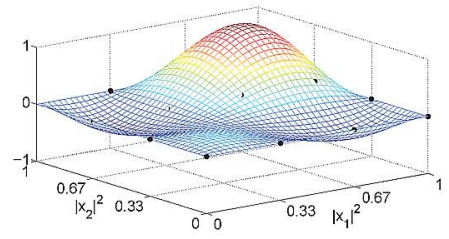

(k)

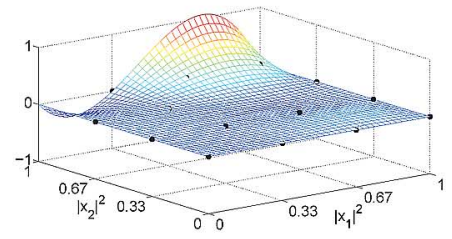

(o)

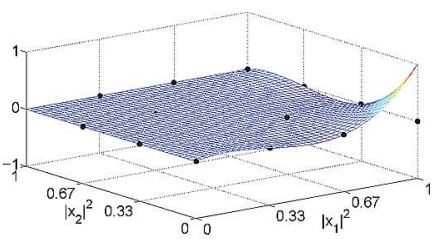

(d)

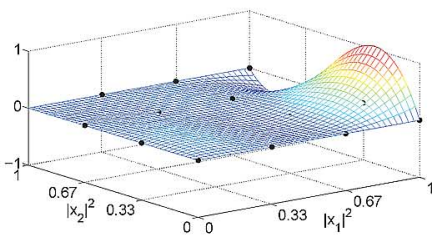

(h)

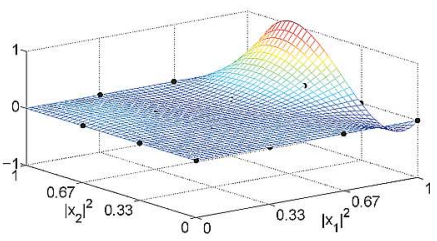

(1)

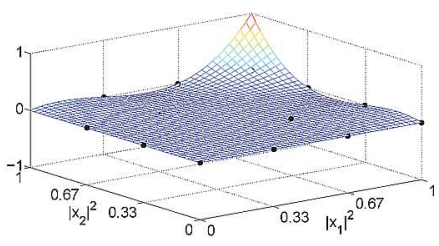

(p)

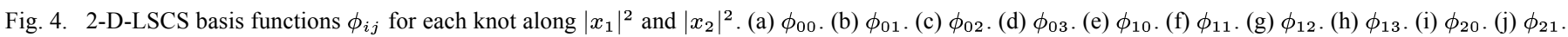
(k) $\phi_{22}$. (l) $\phi_{23}$. (m) $\phi_{30}$. (n) $\phi_{31}$. (o) $\phi_{32}$. (p) $\phi_{33}$.

where $c_{1 i j}^{(m)}$ and $c_{2 i j}^{(m)}$ are the weight coefficients of the 2-D basis for bands 1 and 2, respectively. The basis functions $\phi_{i j}\left(\left|x_{1}\right|^{2},\left|x_{2}\right|^{2}\right)$ are created using 2-D-CSs so that they verify a 2-D Kronecker delta property (8) in which the value of $\phi_{i j}$ at the 2-D knots values $\left(\left|x_{1, u}\right|^{2},\left|x_{2, v}\right|^{2}\right)$ is equal to one when $(i, j)=(u, v)$ and zero for other knots,

$$
\begin{aligned}
\phi_{i j}\left(\left|x_{1, u}\right|^{2},\left|x_{2, v}\right|^{2}\right) \triangleq & \delta_{i, u} \cdot \delta_{j, v} \\
= & \begin{cases}1 & \forall(i, j)=(u, v) \\
0 & \forall \text { other knots }\end{cases} \\
& \forall i \in\left[0, N_{s 1}\right] ; \quad \forall j \in\left[0, N_{s 2}\right] ; \\
& \forall u \in\left[0, N_{s 1}-1\right] ; \quad \forall v \in\left[0, N_{s 2}-1\right] .
\end{aligned}
$$

The $\phi_{i j}$ functions for the case of a four-by-four equally spaced knot grid are plotted in Fig. 4. It can be observed that the $\phi_{i j}$ functions decay rapidly from 1 at $(u, v)$ to 0 at all the other knots over the entire amplitude range. The cubic splines provide continuity up to a second-order derivative between the knots. In this work, the five-by-five knot distribution for both $\left(\left|x_{1, u}\right|^{2}\right.$ and $\left.\left|x_{2, v}\right|^{2}\right)$ is equal to $[0,1 / 4,1 / 2,3 / 4,1]^{2}=[0,1 / 16,1 / 4,9 / 16,1]$ so that the allocation of the $\mathrm{C}$-spline basis degrees of freedom better reflects the data distribution [18]. The knots are indeed not equally spaced because there is a very low probability that both the lower band and upper band signals reach a high level at the same time. Thus there are very few samples that are located above $\left|x_{1}\right|^{2}+\left|x_{2}\right|^{2}=1$ [18]. Taking this data distribution into account, more splines are used near smaller amplitudes and less splines are used close to higher amplitudes.

The 2-D $\phi_{i j}$ functions are synthesized from the 1-D $\phi_{i}$ and $\phi_{j}$ functions along $\left|x_{1}\right|^{2}$ and $\left|x_{2}\right|^{2}$ directions, respectively, as described below.

i) Similar to a Lagrange interpolation polynomial, the 1-D $\phi_{i}\left(\left|x_{1}\right|^{2}\right)$ along $\left|x_{1}\right|^{2}$ is represented using a 1-D C-spline with

$$
\begin{aligned}
\phi_{i}\left(\left|x_{1, u}\right|^{2}\right) \triangleq & \delta_{i, u} \\
= & \begin{cases}1 & \forall i=u \\
0 & \forall \text { other knots }\end{cases} \\
& \forall i \in\left[0, N_{s 1}\right] ; \quad \forall u \in\left[0, N_{s 1}-1\right] .
\end{aligned}
$$

In the knot interval $\left[\left|x_{1, u}\right|^{2},\left|x_{1, u+1}\right|^{2}\right]$, the 1-D $\phi_{i}^{(v)}\left(\left|x_{1}\right|^{2}\right)$ using a 1-D C-spline is then

$$
\begin{aligned}
\phi_{i}^{(u)}\left(\left|x_{1}\right|^{2}\right)= & \sum_{q_{1}=0}^{3} e_{q_{1}}^{(i u)}\left(\left|x_{1}\right|^{2}-\left|x_{1, u}\right|^{2}\right)^{q_{1}} \\
& \forall i \in\left[0, N_{s 1}\right] ; \quad \forall u \in\left[0, N_{s 1}-1\right]
\end{aligned}
$$

where $e_{q_{1}}$ are the coefficients of each spline interpolating between the knots defined in (9) along $\left|x_{1}\right|^{2}$. 
The $\phi_{i}$ functions can be represented in a vector form (11) where each element is computed from a set of splines defined along the entire envelop region

$\Phi_{i}\left(\left|x_{1}\right|^{2}\right)=\left[\phi_{0}\left(\left|x_{1}\right|^{2}\right), \phi_{1}\left(\left|x_{1}\right|^{2}\right), \ldots, \phi_{N_{s 1}}\left(\left|x_{1}\right|^{2}\right)\right]$

ii) Similarly, for the 1-D $\phi_{j}\left(\left|x_{2}\right|^{2}\right)$ along the $\left|x_{2}\right|^{2}$ is represented using a 1-D C-spline with

$$
\begin{aligned}
\phi_{j}\left(\left|x_{2, v}\right|^{2}\right) \triangleq & \delta_{j, v} \\
= & \begin{cases}1 & \forall j=v \\
0 & \forall \text { other knots }\end{cases} \\
& \forall j \in\left[0, N_{s 2}\right] ; \quad \forall v \in\left[0, N_{s 2}-1\right] .
\end{aligned}
$$

In the knot interval $\left[\left|x_{2, v}\right|^{2},\left|x_{2, v+1}\right|^{2}\right]$, the 1-D $\phi_{j}^{(v)}\left(\left|x_{2}\right|^{2}\right)$ using a 1-D C-spline is then

$$
\begin{aligned}
\phi_{j}^{(v)}\left(\left|x_{2}\right|^{2}\right)= & \sum_{q_{2}=0}^{3} e_{q_{2}}^{(j v)}\left(\left|x_{2}\right|^{2}-\left|x_{2, v}\right|^{2}\right)^{q_{2}} \\
& \forall j \in\left[0, N_{s 2}\right], \forall v \in\left[0, N_{s 2}-1\right]
\end{aligned}
$$

where $e_{q_{2}}$ are the coefficients of each spline interpolating between the knots defined in (12) along $\left|x_{2}\right|^{2}$.

The $\phi_{j}$ functions can be represented in the vector form (14) with each column corresponding to a spline along the entire envelop region

$\Phi_{j}\left(\left|x_{2}\right|^{2}\right)=\left[\phi_{0}\left(\left|x_{2}\right|^{2}\right), \phi_{1}\left(\left|x_{2}\right|^{2}\right), \ldots, \phi_{N_{s 2}}\left(\left|x_{2}\right|^{2}\right)\right]$

iii) The tensor product of (10) and (13) generates the 2-D $\phi_{i j}$ functions such that in each of the 2-D knot regions defined by the corners $\left[\left|x_{1, u}\right|^{2},\left|x_{2, v}\right|^{2}\right]$ and $\left[\left|x_{1, u+1}\right|^{2},\left|x_{2, v+1}\right|^{2}\right]$, the 2-D $\phi_{i j}^{(u v)}\left(\left|x_{1}\right|^{2},\left|x_{2}\right|^{2}\right)$ is then

$$
\begin{aligned}
\phi_{i j}^{(u v)} & \left(\left|x_{1}\right|^{2},\left|x_{2}\right|^{2}\right) \\
= & \sum_{q_{1}=0}^{3} \sum_{q_{2}=0}^{3} e_{q_{1}}^{(i u)} \cdot e_{q_{2}}^{(j v)} \cdot\left(\left|x_{1}\right|^{2}-\left|x_{1, u}\right|^{2}\right)^{q_{1}} \\
& \cdot\left(\left|x_{2}\right|^{2}-\left|x_{2, v}\right|^{2}\right)^{q_{2}} \\
& \forall i \in\left[0, N_{s 1}\right] ; \quad \forall j \in\left[0, N_{s 2}\right] ; \\
& \forall u \in\left[0, N_{s 1}-1\right] ; \quad \forall v \in\left[0, N_{s 2}-1\right] .
\end{aligned}
$$

It can be verified from (16) that this new C-spline basis can be used to perfectly model a flat surface without any ripple

$\sum_{i=0}^{N_{s 1}} \sum_{j=0}^{N_{s 2}} \phi_{i j}\left(\left|x_{1}\right|^{2},\left|x_{2}\right|^{2}\right)=1 \quad \forall\left|x_{1}\right|^{2},\left|x_{2}\right|^{2}$.

This guaranties that the model can implement a perfectly constant gain (linear amplifier) without any distortion. Moreover, the locality of the basis is testified at all locations since it is necessary to use all the 2-D basis functions to achieve a constant gain, whereas in the polynomial case, only a single basis with a constant value of 1 is

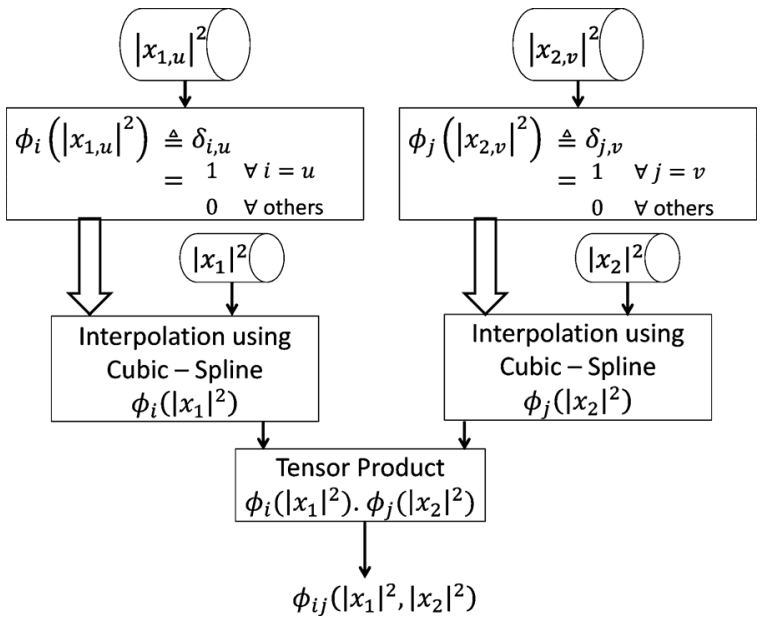

Fig. 5. Flowchart representing the 2-D LSCS basis construction.

used [16]. It can be observed in Fig. 4 that when one basis is active in any specific region, no other basis dominates. The memoryless 2-D function $\phi_{i j}$ can be represented in a vector form similar to a Kronecker product as (17). Although the structure is similar to the polynomial model, it is created using the basis functions generated using C-splines,

$$
\begin{gathered}
\Phi_{i j}\left(\left|x_{1}\right|^{2},\left|x_{2}\right|^{2}\right) \\
=\left\{\left[\phi_{0}\left(\left|x_{1}\right|^{2}\right) \cdot \phi_{0}\left(\left|x_{2}\right|^{2}\right), \phi_{0}\left(\left|x_{1}\right|^{2}\right) \cdot \phi_{1}\left(\left|x_{2}\right|^{2}\right), \ldots,\right.\right. \\
\left.\phi_{0}\left(\left|x_{1}\right|^{2}\right) \cdot \phi_{N_{s 2}}\left(\left|x_{2}\right|^{2}\right)\right] \\
{\left[\phi_{1}\left(\left|x_{1}\right|^{2}\right) \cdot \phi_{0}\left(\left|x_{2}\right|^{2}\right), \phi_{1}\left(\left|x_{1}\right|^{2}\right) \cdot \phi_{1}\left(\left|x_{2}\right|^{2}\right), \ldots,\right.} \\
\left.\phi_{1}\left(\left|x_{1}\right|^{2}\right) \cdot \phi_{N_{s 2}}\left(\left|x_{2}\right|^{2}\right)\right], \ldots, \\
{\left[\phi_{N_{s 1}}\left(\left|x_{1}\right|^{2}\right) \cdot \phi_{0}\left(\left|x_{2}\right|^{2}\right), \phi_{N_{s 1}}\left(\left|x_{1}\right|^{2}\right) \cdot \phi_{1}\left(\left|x_{2}\right|^{2}\right) \ldots,\right.} \\
\left.\left.\phi_{N_{s 1}}\left(\left|x_{1}\right|^{2}\right) \cdot \phi_{N_{s 2}}\left(\left|x_{2}\right|^{2}\right)\right]\right\} \\
=\Phi_{i}\left(\left|x_{1}\right|^{2}\right) \otimes \Phi_{j}\left(\left|x_{2}\right|^{2}\right) .
\end{gathered}
$$

The overall process used in (1) to extract the initial 1-D weights $e_{q_{1}}^{(i u)}$ and $e_{q_{2}}^{(j v)}$ of the 2-D LSCS basis functions and (2) subsequently interpolate the cubic basis functions at any of the input data $\left(\left|x_{1}\right|^{2},\left|x_{2}\right|^{2}\right)$ is represented in the flowchart shown in Fig. 5.

The total number of complex coefficients used in this model are $M \cdot\left(N_{s 1}+1\right) \cdot\left(N_{s 2}+1\right)$, which is reduced by a factor of 16 compared to the 2-D-CS.

Substituting these basis function for the gain in (1) will give the outputs shown in (18),

$$
\begin{aligned}
y_{1}(n)= & \sum_{m=0}^{M-1} x_{1}(n-m) \sum_{i=0}^{N_{s 1}} \sum_{j=0}^{N_{s 2}} c_{1 i j}^{(m)} \\
& \cdot \phi_{i j}\left(\left|x_{1}(n-m)\right|^{2},\left|x_{2}(n-m)\right|^{2}\right) \\
y_{2}(n)= & \sum_{m=0}^{M-1} x_{2}(n-m) \sum_{i=0}^{N_{s 1}} \sum_{j=0}^{N_{s 2}} c_{2 i j}^{(m)} \\
& \cdot \phi_{i j}\left(\left|x_{1}(n-m)\right|^{2},\left|x_{2}(n-m)\right|^{2}\right) .
\end{aligned}
$$

The advantage of the proposed C-spline basis is that the data used for the extraction of the $c_{1 i j}^{(m)}$ and $c_{2 i j}^{(m)}$ coefficients can 


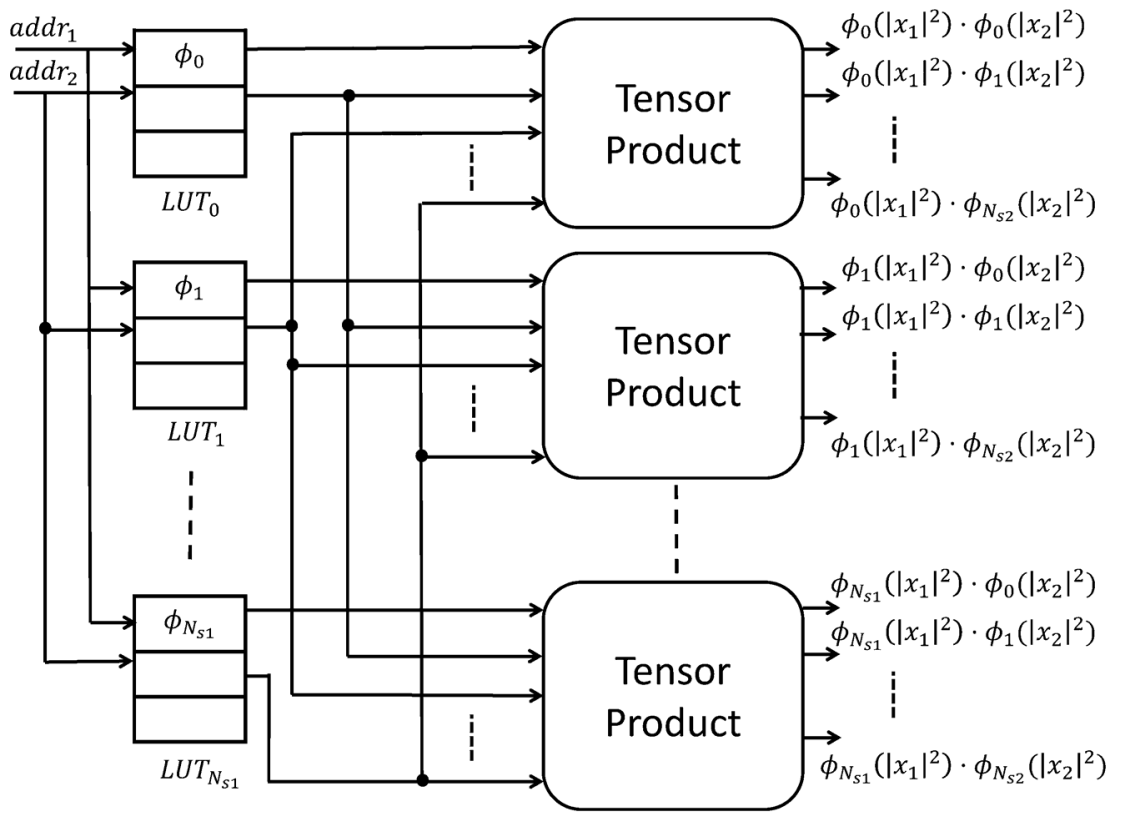

(a)

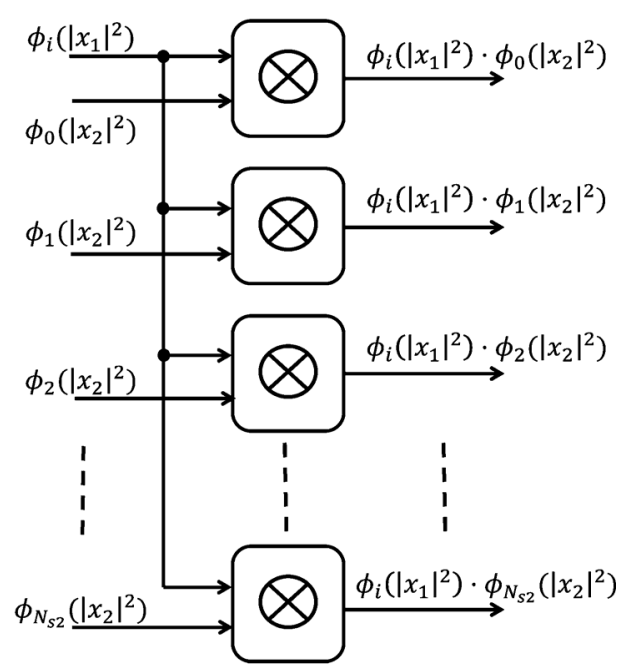

(b)

Fig. 6. Hybrid architecture implementation of 2-D-LSCS and 2-D-MP used in FPGA. (a) 2-D-LSCS basis computation. (b) Tensor product of $\phi_{i}$ and $\phi_{j}$.

now randomly span the complete continuous $\left(\left|x_{1}\right|^{2},\left|x_{2}\right|^{2}\right)$ 2-D space for dual-band PA modeling or $\left(\left|y_{1}\right|^{2},\left|y_{2}\right|^{2}\right)$ 2-D space for dual-band PA linearization. The 2-D-LSCS coefficients can be directly extracted from the measured PA data using the LS method. In the conventional 2-D-CS approach, the data required for the extraction was the discrete $\mathrm{C}$-spline knots, but a direct extraction from the measured PA data was not possible.

2) FPGA Implementation: There are multiple approaches for the implementation of 2-D-LSCS in an FPGA. The merits and limitations of the resulting FPGA architectures are analyzed as follows.

a) The spline coefficients, which are used to construct $\phi_{i j}$ in (18), and weighted coefficients are stored in memory, and (18) are constructed using adders, multipliers, and delay blocks. This kind of implementation requires a lot of resources, thereby limiting the number of splines and also the memory depth of the model that can be implemented for a targeted FPGA. The latency of the model will also be increased due to the large data processing required, leading to an increase in the adaptation time for the model for a particular signal.

b) A 2-D LUT implementation can be used to store the cubic spline basis functions $\phi_{i j}^{(u v)}\left(\left|x_{1}\right|^{2},\left|x_{2}\right|^{2}\right)$ versus discrete values of $\left|x_{1}\right|^{2}$ and $\left|x_{2}\right|^{2}$ [19]. Given the values of $\mid x_{1}(n-$ $m)\left.\right|^{2}$ and $\left|x_{2}(n-m)\right|^{2}$, the gain values can then be extrapolated and used in (1). This implementation is quite general and can be used with other representation techniques extracted using different basis functions. Although this architecture benefits from a reduced latency, it requires a large amount of memory beyond the reach of the conventional FPGA.

c) A hybrid architecture can be used to compromise between the FPGA's resource and memory utilization [11]. The 1-D basis functions are precomputed and stored in memory in 1-D LUTs. The 2-D basis function is computed from the 1-D basis functions inside the FPGA using a reduced number of multipliers. The latency in this hybrid architecture is reduced compared to the latency in a) due to the precomputed LUTs, and the memory in the hybrid architecture is reduced compared to the memory in b) because 1-D functions are used instead of 2-D functions, The weighted coefficients are stored in registers for multiplying with the basis functions to compute the final gain values. Since the LUT can be populated with any desired 1-D basis functions, this architecture can be used by other types of algorithms such as 2-D-MP.

A memoryless gain function is implemented using the hybrid architecture c), as shown in Fig. 6(a). The LUTs shown in Fig. 6(a) are used to store the 1-D basis functions at each knot over the entire amplitude range. The same LUT memory can be used for both the bands since the same basis functions are used, hence all the LUTs have two input and two outputs. A 2-D basis function is calculated using the tensor product of the 1-D basis, as shown in Fig. 6(b). The weighted coefficients are then multiplied with the LUT basis values to obtain the gain value. Once the gain value is computed, the top level architecture is similar to the one shown in Fig. 3(a). Note that the same latency is obtained for all basis functions.

\section{Measurement Results}

The input data is sent from a host computer and stored in the FPGA's double data rate (DDR) memory using MATLAB and a universal serial bus (USB) connection. The downloaded data can be played out to a analog device's mixed-signal DPD (ADI MSDPD) board at the rate of $245.76 \mathrm{MHz}$. The ADI's MSDPD board integrates a complete high-performance RF and mixed-signal transmit and receive chain onto a single board. The DAC in the transmit path is programmed with a sampling 


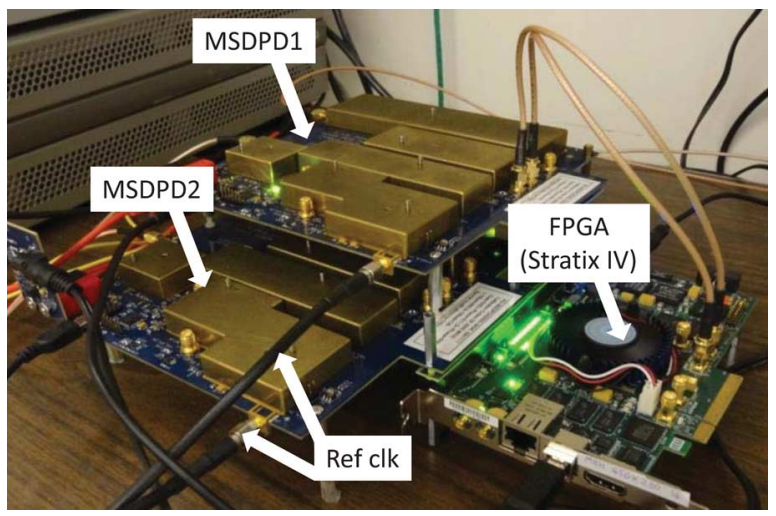

Fig. 7. Measurement Setup for dual-band DPD.

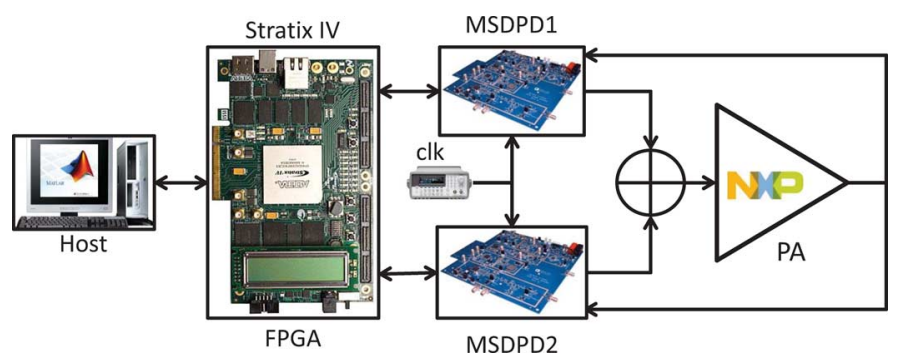

Fig. 8. FPGA testbed used for the experimental verification.

frequency of $983.04 \mathrm{MHz}$, i.e., with an interpolation of 4 . The signal is upconverted using an I/Q modulator and sent to a $10-\mathrm{W}$ NXP PA. The frequency spacing between the bands is around $310 \mathrm{MHz}$ where the lower band and the upper band are centered at 1890 and $2200 \mathrm{MHz}$, respectively. The actual measurement setup for the dual-band DPD is shown in Fig. 7. A reference clock (refclk), as shown in Fig. 8, is used for synchronization between the boards. The output from the PA is filtered and fed back to the observation path on both the boards. The signal is downconverted to a $184.32-\mathrm{MHz}$ IF signal, digitized using 245.76 $\mathrm{MHz}$ and stored in the FPGA's DDR memory. This stored data can be used for further processing such as digital down conversion (DDC) and time alignment [20] using MATLAB. An approximation of the inverse PA model can be extracted by using indirect learning, as in [6], by swapping the input and output in (18) to extract the coefficients for the DPD system.

The 2-D-CS, 2-D-LSCS, and the 2-D-MP methods are compared in this paper. Both the 2-D-CS and 2-D-LSCS methods use a memory depth $M$ of 3 , and $N_{s 1}=N_{s 2}=4$ spline intervals along the $\left|x_{1}\right|^{2}$ and $\left|x_{2}\right|^{2}$ directions. The 2-D-MP model as given in (2) (by swapping $x$ and $y$ ) relies on $M$ of 3 and nonlinearity order $N$ of 7 in order to reach the best possible performance using this approach. The conventional 2-D-CS case relies on the above 2-D-MP model for the initial gain identification.

Two test cases are used for evaluating the efficiency of the developed methods. In the first test case (Case I), a 3c-WCDMA signal with $3.84 \mathrm{MHz}$ of bandwidth in each carrier and LTE signal of $10 \mathrm{MHz}$ are considered. In the second test case (Case II), a 3c-WCDMA signal with $3.84 \mathrm{MHz}$ of bandwidth in each carrier with different seed is considered in each band. The 3c-WCDMA and LTE signals have a high PAPR of 8 and $9 \mathrm{~dB}$, respectively. A two-step experimental procedure is used. In the first experimental step, all the linearization algorithms are extracted and implemented on a host computer (MATLAB) and the predistorted signal data is downloaded onto the DDR memory of the FPGA testbed for transmission to the PA. In the second experimental step, the 2-D-LSCS and 2-D-MP architectures are implemented in the FPGA for the real-time linearization of the dual-band PA. The hybrid architecture is used to design both the 2-D-LSCS and 2-D-MP on the FPGA with an LUT size of 256 for each 1-D basis function. An LUT size of 256 is used to achieve a performance close to the floating point expressions in MATLAB, as shown in Fig. 9. Both the 2-D-LSCS and 2-D-MP implementations exhibit the same DPD latency (14 clocks in the example studied) since the hybrid architecture is independent of the number and type of basis functions selected [11]. The extraction of the basis coefficients is performed on the computer and only the updated basis coefficients $c_{p i j}^{(m)}$ and $d_{p k j}^{(m)}$ of the DPD model are downloaded onto the FPGA.

Using the testbed shown in Fig. 8, the performance of all the methods is established for the same 10-W PA for both test cases (I/II) and for both experimental procedures (MATLAB/FPGA) by training the models on 8192 samples and testing them on a longer frame with 65536 subsequent samples.

The ACPR and NMSE performance results in each test case (I/II) and experimental setup (MATLAB/FPGA) with and without DPD are tabulated in Table I for all methods. In both test cases, the ACPR is calculated at a 5-MHz offset from the signal. It is observed that the signals from the PA output do not fall below the mask requirement of $-45 \mathrm{dBc}$ without DPD. As tabulated in Table I, it can be observed that the best ACPR is obtained using the new proposed 2-D-LSCS approach. An improvement of about $3 \mathrm{~dB}$ in both the ACPR and NSME is observed when compared to other models.

The number of coefficients used in each model is selected so that each model converges within a fraction of a decibel to its optimal ACPR and NMSE values. The value of $N$ is chosen to be 7 based on the performance of the 2-D-MP on the 3c-WCDMA and LTE signal with varying $N$, as shown in Table II, for an extraction dataset of 8192 samples. When $N$ $=8$, the performance of the model based on the ACPR and NMSE was only improved by a fraction of a decibel while the number of coefficients increased by $44 \%$. Thus, a memory depth of 3 is used for all the models . A memory depth of 4 was also verified to yield similar performance as $M=3$ despite the $33 \%$ increase in the number of coefficients. The performance degradation of the 2-D-MP when compared to the 2-D-LSCS is due to the high condition number of the matrix to be inverted. The condition number of the inversion matrix for both the 2-D-MP and 2-D-LSCS is given in Tables III and IV, respectively. The condition number of LSCS remains almost constant as $N s_{1}$ reaches 6 . It can be observed by comparing Tables III and IV that the 2-D-LSCS has a much lower condition number making it resistant to the slight variation in the statistics of the longer input frame. Hence, the performance is less degraded for the 


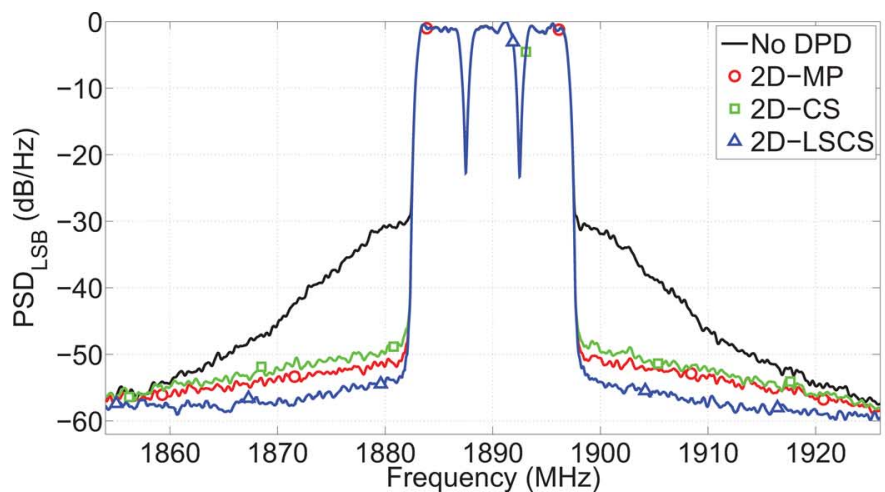

(a)

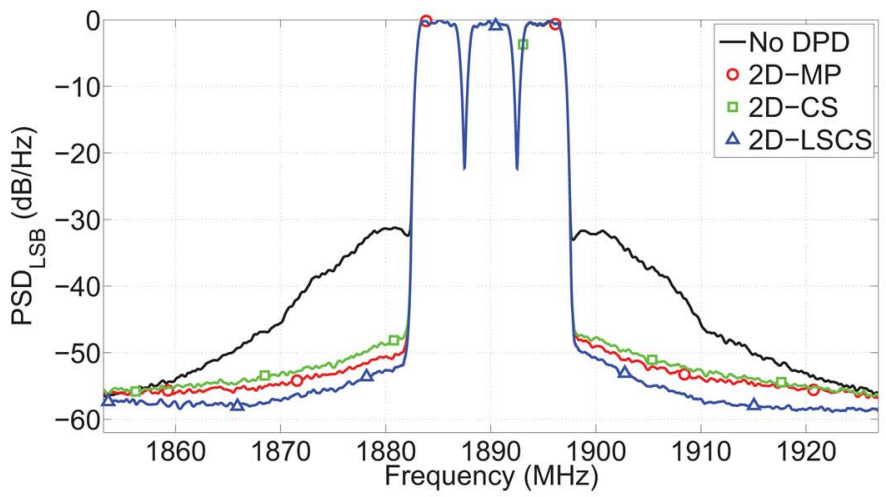

(c)

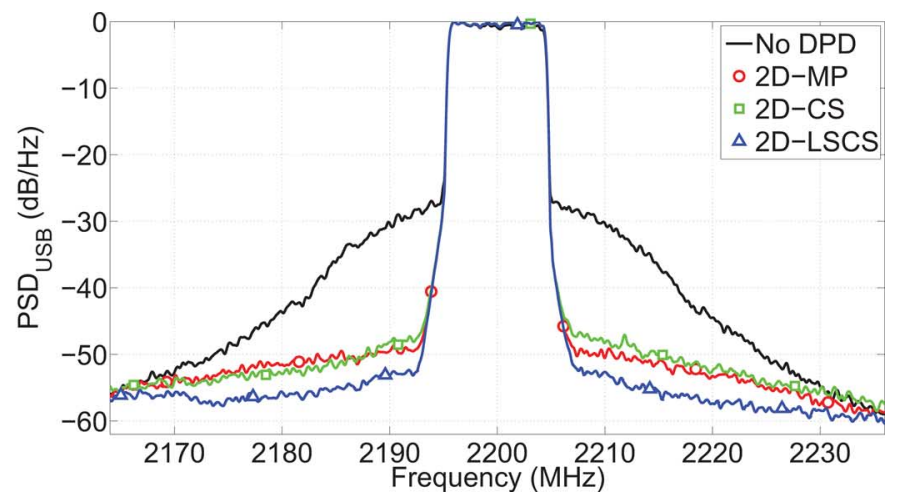

(b)

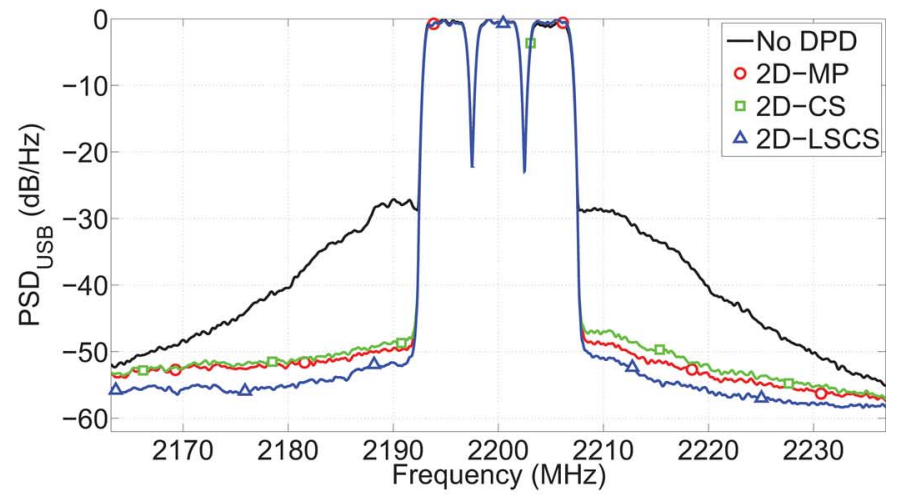

(d)

Fig. 9. Comparison of LSB and USB spectral performance of the 2-D-LSCS, 2-D-CS, and 2-D-MP models for the two test cases using first experimental step. (a) Test Case I: 3-WCDMA (LSB). (b) Test Case I: LTE (10 MHz) (USB). (c) Test Case II: 3-WCDMA (LSB). (d) Test Case II: 3-WCDMA (USB).

TABLE I

NMSE/ACPR FOR BOTH THE BANDS WITH/WITHOUT DPD

\begin{tabular}{|c|c|c|c|c|c|c|c|c|}
\hline & \multicolumn{4}{|c|}{ Test Case I } & \multicolumn{4}{|c|}{ Test Case II } \\
\hline & \multicolumn{2}{|c|}{ 3-WCDMA (LSB) } & \multicolumn{2}{|c|}{ LTE(10 MHz) (USB) } & \multicolumn{2}{|c|}{ 3-WCDMA (LSB) } & \multicolumn{2}{|c|}{ 3-WCDMA (USB) } \\
\hline & NMSE (dB) & $\begin{array}{l}\text { ACPR }(\mathrm{dBc}) \\
@-/+5 \mathrm{MHz}\end{array}$ & NMSE (dB) & $\begin{array}{l}\text { ACPR }(\mathrm{dBc}) \\
@-/+5 \mathrm{MHz}\end{array}$ & NMSE (dB) & $\begin{array}{l}\text { ACPR (dBc) } \\
@-/+5 \mathrm{MHz}\end{array}$ & NMSE (dB) & $\begin{array}{l}\text { ACPR }(\mathrm{dBc}) \\
@-/+5 \mathrm{MHz}\end{array}$ \\
\hline 2D MP & -40.68 & $-50.21 /-49.78$ & -41.25 & $-49.11 /-49.71$ & -41.37 & $-49.77 /-48.10$ & -41.40 & $-48.94 /-48.11$ \\
\hline 2D-CS & -39.19 & $-48.12 /-48.03$ & -37.86 & $-48.65 /-47.88$ & -37.05 & $-47.70 /-47.17$ & -39.47 & $-47.92 /-46.47$ \\
\hline FPGA: 2D-MP & -39.30 & $-49.80 /-49.17$ & -38.21 & $-49.65 /-49.51$ & -42.00 & $-49.86 /-48.47$ & -39.90 & $-48.58 /-48.08$ \\
\hline FPGA: 2D-LSCS & -42.56 & $-52.54 /-52.26$ & -41.59 & $-52.71 /-53.26$ & -42.46 & $-52.17 /-50.50$ & -41.65 & $-51.57 /-50.24$ \\
\hline
\end{tabular}

TABLE II

PERFormance COMPARISON OF 2-D-MP With VARYING NONLINEARITY ORDER AND 2-D-LSCS ON A SHORT FRAME (8192 SAMPLES)

\begin{tabular}{|c|c|c|c|c|}
\hline \multirow{2}{*}{$\begin{array}{c}\text { Model } \\
\text { Order }\end{array}$} & \multicolumn{2}{|c|}{ ACPR $@ 5 \mathrm{MHz}$} & \multicolumn{2}{c|}{ NMSE } \\
\cline { 2 - 5 } & LSB & USB & LSB & USB \\
\hline 2D-MP (N=5) & $-48.9 /-50.9$ & $-49.1 /-48.0$ & -40.0 & -40.3 \\
\hline 2D-MP (N=6) & $-51.3 /-53.3$ & $-51.4 /-50.4$ & -39.0 & -42.5 \\
\hline 2D-MP (N=7) & $-54.0 /-56.6$ & $-53.3 /-53.6$ & -42.9 & -40.8 \\
\hline 2D-LSCS & $-53.8 /-55.7$ & $-54.0 /-54.5$ & -43.0 & -44.3 \\
\hline
\end{tabular}

2-D-LSCS than for the 2-D-MP, when the model is trained on a shorter frame and validated on a longer frame.
TABLE III

CONDITION NUMBER FOR 2-D-MP WITH VARYING NONLINEARITY ORDER (N)

\begin{tabular}{|c|c|c|}
\hline \multirow{2}{*}{$\begin{array}{c}\text { Model } \\
(\mathrm{N})\end{array}$} & \multicolumn{2}{|c|}{ Condition Number } \\
\cline { 2 - 3 } & LSB & USB \\
\hline 5 & $1.36 \times 10^{5}$ & $1.23 \times 10^{5}$ \\
\hline 6 & $1.26 \times 10^{6}$ & $1.09 \times 10^{6}$ \\
\hline 7 & $4.61 \times 10^{6}$ & $4.09 \times 10^{6}$ \\
\hline
\end{tabular}

The lower sideband (LSB) and upper sideband (USB) spectra obtained with the new proposed 2-D-LSCS (blue line (in online version) and triangles), the conventional 2-D-CS (green line (in online version) and squares) and the reference 2-D-MP 


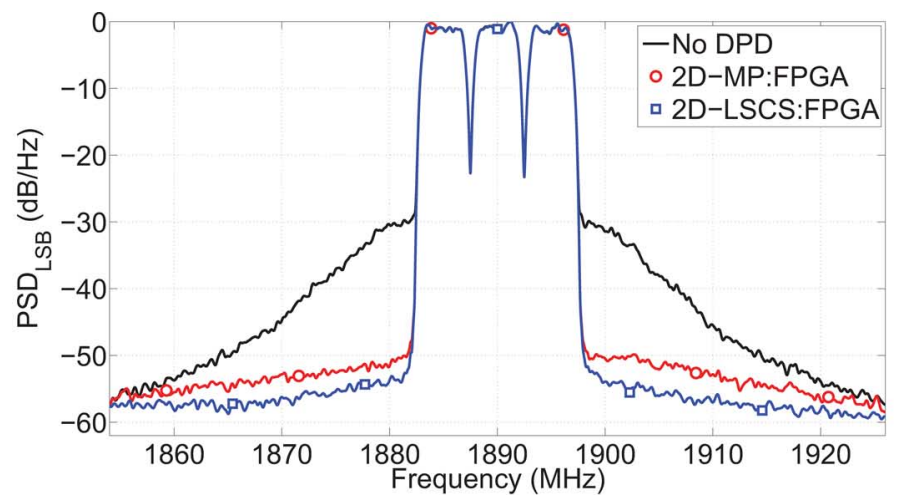

(a)

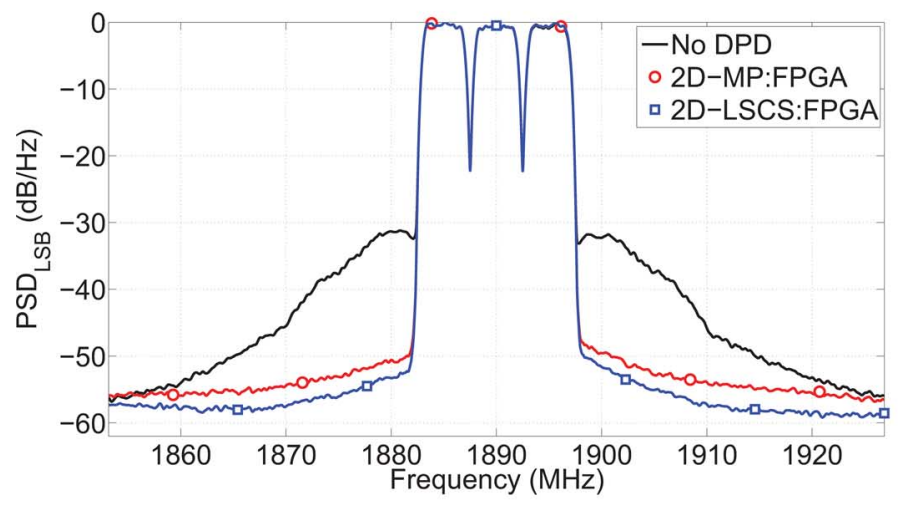

(c)

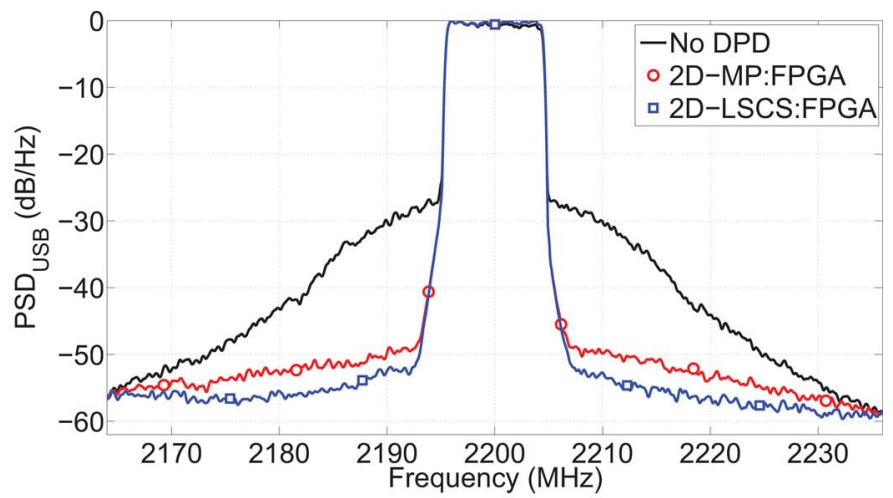

(b)

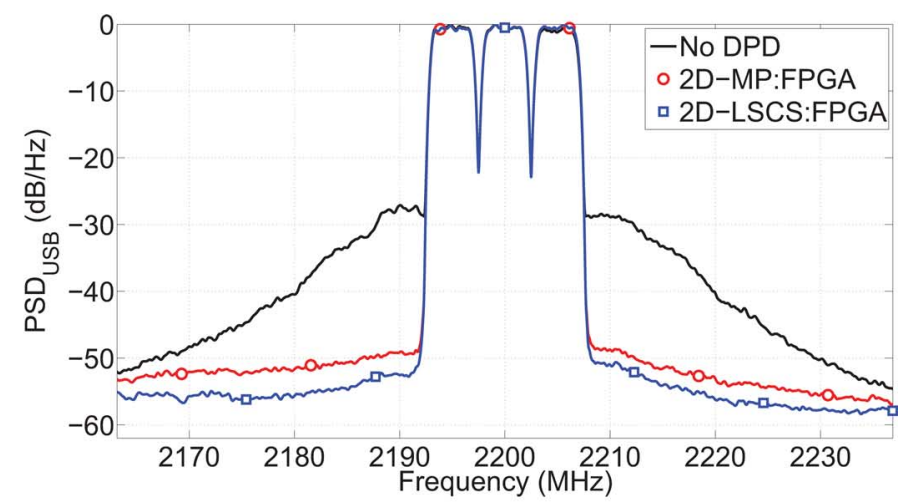

(d)

Fig. 10. Comparison of the LSB and USB spectral performance of the 2-D-LSCS and 2-D-MP models for the two test cases using second experimental step with a hybrid real-time architecture inside the FPGA. (a) Test Case I: 3-WCDMA (LSB). (b) Test Case I: LTE (10 MHz) (USB). (c) Test Case II: 3-WCDMA (LSB). (d) Test Case II: 3-WCDMA (USB).

TABLE IV

CONDITION NUMBER FOR 2-D-LSCS WITH VARYING NUMBER OF SPLINES IN EACH AXIS

\begin{tabular}{|c|c|c|}
\hline \multirow{2}{*}{$\begin{array}{c}\text { Model } \\
\left(N s_{1}\right)\end{array}$} & \multicolumn{2}{|c|}{ Condition Number } \\
\cline { 2 - 3 } & LSB & USB \\
\hline 3 & $1.29 \times 10^{4}$ & $1.41 \times 10^{4}$ \\
\hline 4 & $1.20 \times 10^{4}$ & $1.37 \times 10^{4}$ \\
\hline 5 & $1.81 \times 10^{4}$ & $1.98 \times 10^{4}$ \\
\hline 6 & $4.19 \times 10^{4}$ & $4.98 \times 10^{4}$ \\
\hline
\end{tabular}

(red line (in online version) and circles) models for the two above test cases I and II are compared in Fig. 9(a)-(d) when both the extraction and the implementation are performed on a computer and the resulting predistorted data is downloaded onto the FPGA testbed to be transmitted to the PA. The PA output without DPD is shown using a black line for reference. In Fig. 10(a)-(d), the performance of 2-D-LSCS (blue line (in online version) and rectangles) and 2-D-MP (red line (in online version) and circles) is compared for the case where the predistortion linearization of the dual-band waveforms is performed in real-time inside the FPGA using the hybrid architecture in Fig. 6. For both the offline MATLAB predistortion in Fig. 9 and the real-time FPGA predistortion in Fig. 10, the 2-D-LSCS is observed to provide the best performance in both the bands with up to $3-\mathrm{dB}$ reduction in sideband spectral density compared to the reference 2-D-MP model.
TABLE V

Total Number of Coefficients Used by Each Model

\begin{tabular}{|c|c|c|}
\hline \multirow{2}{*}{ 2D Model } & \multicolumn{2}{|c|}{ Number of Complex Coefficients } \\
\cline { 2 - 3 } & General & Test Case \\
\hline 2D-MP & $M \cdot N \cdot(N+1)$ & 168 \\
\hline 2D-CS & $2 \cdot M \cdot N_{s 1} \cdot N_{s 2} \cdot 16$ & 1536 \\
\hline 2D-LSCS & $2 \cdot M \cdot\left(N_{s 1}+1\right) \cdot\left(N_{s 2}+1\right)$ & 150 \\
\hline
\end{tabular}

The number of coefficients required for each model to achieve its best performance is given in Table V. It is observed that 2-D-LSCS requires fewer coefficients while achieving a better performance when compared to other models. The overall extraction time including both the matrix setup and its inversion is also shown in Table VI. The number of floating point operations per second (FLOPS) used in the inversion of the matrix [21] for the coefficient extraction is reduced as shown in Table VI. The extraction of the 2-D-LSCS is thus substantially faster than that of the 2-D-MP case. This is due to the fact that the 2-D-LSCS relies on piece-wise polynomials of reduced order and requires a smaller number of basis functions.

The reduction of the logic resources used for the real-time implementation of the linearization algorithm in the FPGA is also important for yielding a low-cost implementation. The hybrid implementation in Fig. 6 is used to implement 2-D-LSCS and 2-D-MP inside the FPGA because of its balanced usage of multipliers and memory [11]. It can be verified 
TABLE VI

COMPARISON OF THE DPD EXTRACTION TIME AND REAL-TIME DSP RESOURCE UTILIZATION

\begin{tabular}{|c|c|c|c|c|c|}
\hline \multirow{2}{*}{$\begin{array}{c}\text { Basis } \\
\text { Function }\end{array}$} & \multicolumn{2}{|c|}{ DPD Extraction } & \multicolumn{3}{|c|}{ DSP Resource } \\
\cline { 2 - 6 } & $\begin{array}{c}\text { Time } \\
(\mathrm{sec})\end{array}$ & $\begin{array}{c}\text { FLOPS } \\
\text { (Giga) }\end{array}$ & $\otimes$ & $\oplus$ & $\begin{array}{c}\text { Memory } \\
(\mathrm{KB})\end{array}$ \\
\hline 2D-MP & 0.62 & 9.2 & 379 & 346 & 3 \\
\hline 2D-LSCS & 0.5 & 8.2 & 355 & 310 & 2.5 \\
\hline
\end{tabular}

TABLE VII

FPGA RESOURCE UTILIZATION

\begin{tabular}{|c|c|c|c|c|c|}
\hline Mode & Logic Utilization & ALUT & $\begin{array}{c}\text { M9K } \\
\text { Blocks }\end{array}$ & Registers & $\begin{array}{c}\text { Signed } \\
\text { Multipliers }\end{array}$ \\
\hline MP & 12511 & 6253 & 6 & 13666 & 392 \\
\hline LSCS & 10948 & 5528 & 5 & 11469 & 378 \\
\hline
\end{tabular}

from Table VI that the total number of real multipliers, adders, and LUT memory used by the 2-D-LSCS model is less than that used by the 2-D-MP model.

Although both of the bands use the same 1-D basis function, the basis values must be obtained based on the envelope square of each band. A single dual-port RAM (LUT) is then sufficient to store each of the 1-D $\phi$ functions for both the bands, as shown in Fig. 6(a). The LUTs used to store the 1-D basis function are synthesized in the FPGA using Stratix IV M9K blocks, which can be instantiated as dual-port memories. The multipliers and adders are used from the library of parametrized modules (LPM) available from the Altera intellectual property (IP) suite. The LUT values are generated offline to initialize the M9K blocks and are never updated later on. The FPGA resources used by the 2-D-LSCS and 2-D-MP are tabulated in Table VII for comparison. Most of the combinational logic is implemented inside an FPGA using adaptive lookup tables (ALUTs). The Logic Utilization parameter provides a good representation of all the resources used inside an FPGA for the real-time calculation. The Logic Utilization parameter in Table VII confirms that 2-D-LSCS utilizes less FPGA resources than the polynomial representation.

\section{CONCLUSION}

In this paper, a dual-band DPD system has been implemented using a new 2-D-CS basis (2-D-LSCS) and its performance has been compared to the conventional 2-D-CS and 2-D-MP models. Unlike the 2-D-CS, this method can be directly extracted from the measured data using the LS method without depending on any other model. Besides retaining the nonoscillatory advantage of a low-order piece-polynomial representation, the new 2-D-CS basis also exhibits a local-focus (2-D Kronecker delta property) and relies on a nonuniform knot distribution to better account for the data distribution. All these attributes are engineered into the model to increase the extraction robustness.

The new LSCS approach can be implemented using a hybrid architecture [11], which stores the 1-D basis functions in LUTs and numerically computes the 2-D basis functions using tensor product multipliers. This architecture is implemented for both the 2-D-LSCS and 2-D-MPs to optimize the management of the FPGA DSP resources and reduce the real-time latency.
This DPD can be easily implemented on an FPGA or application specific integrated circuits that are used to linearize the PA. The new 2-D-LSCS achieved the best performance with fewer FPGA resources, fewer basis coefficients, and reduced extraction time when compared to conventional 2-D-CS and 2-D-MP models for the two dual-band scenarios examined. The decrease in the number of basis coefficients and basis functions reduces the computational resource utilization of the FPGA. The decrease in the number of coefficients reduces the computational resource utilization of the FPGA, and furthermore reduces the extraction time of the model. The measurement results show that even though 2-D-LSCS utilizes less real-time DSP/FPGA resources and requires less extraction time, a performance improvement in the NMSE and ACPR by up to $3 \mathrm{~dB}$ is still observed compared to the reference 2-D-MP model. The improvement in performance of the 2-D-LSCS method over the 2-D-MP is validated during the linearization of subsequent longer dataset for which the dual-band linearization system was not trained. This increased performance for the new 2-D-LSCS extraction originates from the engineered characteristics of the new proposed 2-D-CS basis.

\section{ACKNOWLEDGMENT}

The authors would like to thank Analog Devices Inc., Wilmington, MA, USA, and NXP Semiconductors, Smithfield, RI, USA, for donating the mixed signal digital pre-distortion (MSDPD) system boards and the PAs, respectively, which are used in this study.

The authors would like to thank the Altera Corporation-Wireless Systems Solutions Group, Buckinghamshire, U.K., for their technical support in this project and the donation of the Stratix IV field-programmable gate array (FPGA).

The authors would also like to thank K. Berndsen, GatesAir, Mason, $\mathrm{OH}, \mathrm{USA}$, for his valuable suggestions. The authors are grateful to the anonymous reviewers for inspiring suggestions, which greatly improved the quality of this paper.

\section{REFERENCES}

[1] L. Ding, Z. Yang, and H. Gandhi, "Concurrent dual-band digital predistortion," in IEEE MTT-S Int. Microw. Symp. Dig., 2012, pp. 1-3.

[2] R. Liu, D. Schreurs, W. De Raedt, F. Vanaverbeke, and R. Mertens, "Concurrent dual-band power amplifier with different operation modes," in IEEE MTT-S Int. Microw. Symp. Dig., Jun. 2011, pp. 1-4.

[3] P. Roblin et al., "Frequency-selective predistortion linearization of RF power amplifiers," IEEE Trans. Microw. Theory Techn., vol. 56, no. 1, pp. 65-76, Jan. 2008.

[4] R. Braithwaite, "Digital predistortion of a power amplifier for signals comprising widely spaced carriers," in 78th ARFTG Microw. Meas. Symp., 2011, pp. 1-4.

[5] N. Naraharisetti, C. Quindroit, P. Roblin, S. Gheitanchi, V. Mauer, and M. Fitton, "2D cubic spline implementation for concurrent dual-band system," in IEEE MTT-S Int. Microw. Symp. Dig., Jun. 2013, pp. 1-4.

[6] S. Bassam, M. Helaoui, and F. Ghannouchi, "2-D digital predistortion (2-D-DPD) architecture for concurrent dual-band transmitters," IEEE Trans. Microw. Theory Techn., vol. 59, no. 10, pp. 2547-2553, Oct. 2011.

[7] A. Kwan, S. Bassam, M. Helaoui, and F. Ghannouchi, "Concurrent dual band digital predistortion using look up tables with variable depths," in IEEE Power Amplifiers Wireless Radio Appl. Top. Conf., 2013, pp. 25-27.

[8] C. Quindroit, N. Naraharisetti, P. Roblin, S. Gheitanchi, V. Mauer, and M. Fitton, "2D forward twin nonlinear two-box model for concurrent dual-band digital predistortion," in IEEE Power Amplifiers Wireless Radio Appl. Top. Conf., Jan. 2014, pp. 1-3. 
[9] N. Naraharisetti, P. Roblin, C. Quindroit, M. Rawat, and S. Gheitanchi, "2d quasi exact inverse of PA model in digital predistorter for concurrent dual-band system," in IEEE 15th Annu. Wireless Microw. Technol. Conf., Jun. 2014, pp. 1-4.

[10] N. Safari, N. Holte, and T. Roste, "Digital predistortion of power amplifiers based on spline approximations of the amplifier characteristics," in IEEE 66th Veh. Technol. Conf., 2007, pp. 2075-2080.

[11] C. Quindroit, N. Naraharisetti, P. Roblin, S. Gheitanchi, V. Mauer, and M. Fitton, "FPGA implementation of orthogonal 2D digital predistortion system for concurrent dual-band power amplifiers based on time-division multiplexing," IEEE Trans. Microw. Theory Techn., vol. 61, no. 12, pp. 4591-4599, Dec. 2013.

[12] A. Hussein, V. Bohara, and O. Venard, "Two-dimensional memory selective polynomial model for digital predistortion," in IEEE 10th Int. New Circuits Syst. Conf., 2012, pp. 469-472.

[13] J. Moon, P. Saad, J. Son, C. Fager, and B. Kim, "2-D enhanced hammerstein behavior model for concurrent dual-band power amplifiers," in 42nd Eur. Microw. Conf., 2012, pp. 1249-1252.

[14] C. Quindroit, N. Naraharisetti, P. Roblin, S. Gheitanchi, V. Mauer, and M. Fitton, "Concurrent dual-band digital predistortion for power amplifier based on orthogonal polynomials," in IEEE MTT-S Int. Microw. Symp. Dig., Jun. 2013, pp. 1-4.

[15] C. DeBoor, A Practical Guide to Splines, 3rd ed. Berlin, Germany: Springer-Verlag, 1978, ch. 3 and 4, pp. 31-45.

[16] F. Barradas, T. Cunha, P. Lavrador, and J. Pedro, "Higher locality non-linear basis functions of volterra series based models to improve extraction conditioning," in IEEE MTT-S Int. Microw. Symp. Dig., Jun. 2014, pp. 1-4.

[17] X. Wu, N. Zheng, X. Yang, J. Shi, and H. Chen, "A spline-based hammerstein predistortion for $3 \mathrm{G}$ power amplifiers with hard nonlinearities," in 2nd Int. Future Comput. Commun. Conf., 2010, vol. 3, pp. V3-741-V3-745.

[18] P. Roblin, N. Naraharisetti, C. Quindroit, S. Gheitanchi, V. Mauer, and M. Fitton, "2D multisine mapping for robust 2 band PA modeling and 2D predistorter extraction," in 81 st ARFTG Microw. Meas. Conf., Jun. 2013, pp. 1-3.

[19] L. Guan and A. Zhu, "Low-cost FPGA implementation of Volterra series-based digital predistorter for RF power amplifiers," IEEE Trans. Microw. Theory Techn., vol. 58, no. 4, pp. 866-872, Apr. 2010.

[20] S. Boumaiza, M. Helaoui, O. Hammi, T. Liu, and F. Ghannouchi, "Systematic and adaptive characterization approach for behavior modeling and correction of dynamic nonlinear transmitters," IEEE Trans. Instrum. Meas., vol. 56, no. 6, pp. 2203-2211, Jun. 2007.

[21] M. Arakawa, "Computational workloads for commonly used signal processing kernels," MIT Lincoln Lab., Cambridge, MA, USA, Project Rep. SPR-9, 2006.

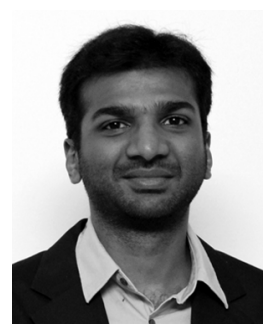

Naveen Naraharisetti (M'13) received the Bachelor of Technology degree in electronics and communications engineering from Acharya Nagarjuna University, Andhra Pradesh, India, in 2005, the M.S degree in electrical and computer engineering from the University of Michigan, Dearborn, MI, USA in 2008, and the M.S and Ph.D degrees in electrical and computer engineering from The Ohio State University, Columbus, OH, USA in 2014.

He is currently a Senior Electrical Engineer with GatesAir Inc., Mason, OH, USA. His research inter- ests are concurrent multiband digital predistortion and crest factor reduction for power amplifiers with field-programmable gate-array (FPGA) implementation.

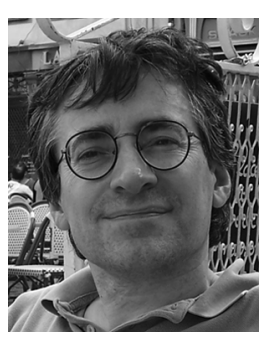

Patrick Roblin (M'85-SM'14) was born in Paris, France, in September 1958. He received the Maitrise de Physics degree from Louis Pasteur University, Strasbourg, France, in 1980, and the M.S. and D.Sc. degrees in electrical engineering from Washington University, St. Louis, MO, USA, in 1982 and 1984, respectively.

In 1984, he joined the Department of Electrical Engineering, The Ohio State University (OSU), Columbus, $\mathrm{OH}, \mathrm{USA}$, as an Assistant Professor. He is currently a Professor with OSU. He is the lead author of the textbooks High-Speed Heterostructure and Devices (Cambridge Univ. Press, 2002) and Nonlinear RF Circuits and Nonlinear Vector Network Analyzers (Cambridge Univ. Press, 2011). With OSU, he is the Founder of the NonLinear RF Research Laboratory and has developed two educational $\mathrm{RF} /$ microwave laboratories and associated curriculum for training both undergraduate and graduate students. His current research interests include the measurement, modeling, design, and linearization of nonlinear RF devices and circuits such as oscillators, mixers, and power amplifiers.

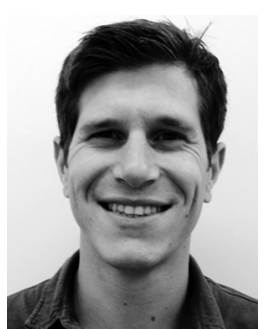

Christophe Quindroit was born in Corbeil-Essonnes, France, in October 1982. He received the M.Tech. and M.S. degrees in electronics from the Ecole Polytechnique de l'Universite de Nantes, Nantes, France, in 2005, and the Ph.D. degree in electronics from XLIM, University of Limoges, Limoges, France, in 2010.

He was a Project Engineer with Alcatel-Lucent, Lannion, France. From 2012 to 2014, he was a Research Engineer with The Ohio State University, Columbus, OH, USA. He is currently a Digital Radio Engineer with LPA Concepts, Martillac, France. His research interests include analog system-level modeling, power amplifier (PA) linearization techniques, and field-programmable gate-array (FPGA) implementation.

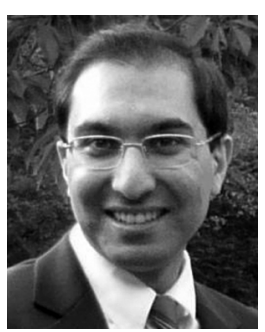

Shahin Gheitanchi (M'04) received the M.Sc. degree in digital communications and Ph.D. degree from the University of Sussex, Sussex, U.K., in 2004 and 2009, respectively.

$\mathrm{He}$ is currently with the Wireless Systems Solutions Group, Altera Europe Limited, Buckinghamshire, U.K. He has authored or coauthored a number of international publications. He holds numerous patents. His research interests include multicarrier multiple-access techniques, adaptive real-time signal processing, crest factor reduction, adaptive digital pre-distortion, application of biologically inspired artificial intelligence for optimization, and heterogeneous multistandard networks. 